\title{
Aktuelles Schlagwort
}

Die Vollzeitpflege bildet eine Maßnahme der stationären Jugendhilfe, die mit besonderen Herausforderungen für alle Beteiligten - Pflegekind, Herkunftsfamilie und Pflegefamilie - einhergeht. Dabei sind die Gründe, die zur Inpflegegabe eines Kindes führen, vielfältig. Relativ häufig werden die psychische Erkrankung eines Erziehungsberechtigten, ebenso wie Alkohol- oder Drogenprobleme als Ursachen angeführt, seltener auch Tod oder Inhaftierung eines Elternteils. Welchen Problemen die betroffenen Familien auch immer ausgesetzt sind: Sie führen meist dazu, dass die Eltern der adäquaten Betreuung und Versorgung ihrer Kinder nicht mehr nachkommen können, was schließlich die Fremdunterbringung erforderlich macht. Was die Zahl der Kinder und Jugendlichen anbelangt, die derzeit in Deutschland in einer Pflegefamilie leben, so liegen keine verlässlichen Angaben vor. Schätzungen variieren zwischen 40.000 und 135.000, wobei das Verhältnis Fremdvollpflege/Verwandtenpflege unbekannt ist. Unklarheiten bestehen nicht nur hinsichtlich der Zahl der Pflegschaften in Deutschland. Generell weist die Forschungsliteratur zum Pflegekinderwesen deutliche Lücken auf. So ist beispielsweise über die Entwicklung junger Erwachsener, die einen Teil ihrer Kindheit und Jugend in einer Pflegefamilie verbracht haben, bislang wenig bekannt: Wie zufrieden sind sie mit ihrem Leben? Wie beurteilen sie rückblickend die Unterbringung in einer Pflegefamilie? Mit welchen Schwierigkeiten hatten sie zu kämpfen? Wofür sind sie ihren Pflegeeltern dankbar? Diesen und ähnlichen Fragen wurde im Rahmen einer Untersuchung des Pflegekinderdiensts des Stadtjugendamts München nachgegangen. Dazu wurden 33 ehemalige, nun erwachsene Pflegekinder, die durch den Münchner Pflegekinderdienst vermittelt und/oder betreut worden waren, interviewt. Die teils sehr berührenden Schilderungen verdeutlichen zentrale Herausforderungen, mit denen das Aufwachsen in einer Pflegefamilie verbunden ist. Einen wichtigen Aspekt bildet sicherlich das Streben nach Normalität oder - anders ausgedrückt - das Hadern mit dem Anderssein, also der Erkenntnis, anders aufzuwachsen als Freunde und Mitschüler, anderen Familienverhältnissen zu entstammen. Dieses Bewusstsein geht häufig mit einem als unangenehm erlebten Erklärungsnotstand einher. Etwa, wenn die Schulkameraden wissen möchten, warum man nicht bei den leiblichen Eltern aufwächst, wenn sich jemand erkundigt, weshalb man nicht den Namen der Eltern trägt oder wenn Bekannte erstaunt ausrufen, dass man dem Rest der Familie ja gar nicht ähnlich sehe. Gleichzeitig sind es oftmals die ganz alltäglichen, vielleicht gerade die für andere Kinder so „normalen“ Dinge, die von einigen Befragten rückblickend besonders wertschätzend betont werden. So spielt die mit den Pflegeeltern und -geschwistern verbrachte Zeit eine essentielle Rolle - sei es das allabendliche gemeinsame Vorlesen vor dem Zubettgehen oder die im Familienkreis eingenommene Mahlzeit. Diese Aktivitäten und alltäglichen Routinen tragen sicherlich maßgeblich dazu bei, dass sich bei den Pflegekindern ein Gefühl der Zugehörigkeit entwickeln kann, ein Gefühl des Aufgehoben- und Angenommenseins. Dieses Zugehörigkeitsgefühl scheint - ebenso wie die Konfrontation mit dem Anderssein und dem Streben nach Normalität - einen Kern der Schwierigkeiten zu berühren, mit denen Pflegekinder zu kämpfen haben. Weitere Anforderungen, wie der Umgang mit der Etikettierung als Pflegekind oder die Suche nach den eigenen biographischen Wurzeln, ließen sich dem noch hinzufügen. Doch nicht nur die Pflegekinder und ihre häufig sozial und ökonomisch benachteiligten Herkunftsfamilien haben vielfältige Herausforderungen zu bewältigen. Auch sollte die Rolle der Pflegefamilien nicht vergessen werden, die sich mit der oftmals konfliktreichen Vorgeschichte des Pflegekindes auseinandersetzen müssen und die durch die Aufnahme des Kindes u.a. ihren Status als Privatfamilie verlieren und in Teilen zu einer Institution des Jugendamts werden.

Birgit Heppt 